\title{
On Dependence of Sets of Functions on the Mean Value of their Elements
}

\section{U. Raitums}

University of Latvia, Institute of Mathematics and Computer Science 29 Rainis boulevard, LV-1459 Riga, Latvia

E-mail: uldis.raitums@lumii.lv

Received September 11, 2008; revised December 23, 2008; published online February 25, 2009

Abstract. The paper considers, for a given closed bounded set $M \subset \mathbb{R}^{m}$ and $K=$ $(0,1)^{n} \subset \mathbb{R}^{n}$, the set $\mathcal{M}=\left\{h \in L_{2}\left(K ; \mathbb{R}^{m}\right) \mid h(x) \in M\right.$ a.e. $\left.x \in K\right\}$ and its subsets

$$
\mathcal{M}(\hat{h})=\left\{h \in \mathcal{M} \mid \int_{K} h(x) d x=\hat{h}\right\} .
$$

It is shown that, if a sequence $\left\{\hat{h}_{k}\right\} \subset c o M$ converges to an element $h_{k} \in \mathcal{M}\left(\hat{h}_{k}\right)$ there is $h_{k}^{\prime} \in \mathcal{M}\left(\hat{h}_{0}\right)$ such that $h_{k}^{\prime}-h_{k} \rightarrow 0$ as $k \rightarrow \infty$. If, in addition, the set $M$ is finite or $M$ is the convex hull of a finite set of elements, then the multivalued mapping $\hat{h} \rightarrow \mathcal{M}(\hat{h})$ is lower semicontinuous on $\operatorname{co} M$.

Key words: multivalued mapping, subsets of functions with fixed mean value, continuous dependence.

\section{Introduction}

Most sets of admissible control functions in the theory of optimal control are given as sets of measurable functions with values from a given set: for a given reference domain $Q \subset \mathbb{R}^{n}$ and a given set $M \subset \mathbb{R}^{m}$ the set of admissible controls is defined as

$$
\mathcal{M}=\{h \text { is measurable in } Q \mid h(x) \in M \text { a.e. } x \in Q\} .
$$

Here $n$ and $m$ are arbitrary fixed positive integers.

Provided that $Q$ is a bounded domain and $M$ is a bounded and closed set, the set $\mathcal{M}$ can be split as $\mathcal{M}=\bigcup_{\hat{h} \in \operatorname{co} M} \mathcal{M}(\hat{h})$, where

$$
\mathcal{M}(\hat{h}):=\left\{h \text { measurable, } h(x) \in M \text { a.e. } x \in Q, \quad \frac{1}{|Q|} \int_{Q} h(x) d x=\hat{h}\right\} .
$$

Here by $\operatorname{co} A$ we denote the convex hull of the set $A$ and by $|Q|$ we denote the Lebesgue measure of the set $Q \subset \mathbb{R}^{n}$. 
Such a representation of $\mathcal{M}$ is useful when weak limits of sequences of control functions are involved, especially in procedures of relaxation via convexification, see, for instance, Warga [4]. Analogous splitting is used in the homogenization theory defining the so-called $G_{\theta}$-closures, see, for instance, Milton [2]. The corresponding relaxation procedures often involve the evaluation of integrals (over the periodicity cell $K=(0,1)^{n}$ ) of the kind

$$
I(\hat{h})=\inf _{h \in \mathcal{M}(\hat{h})} \int_{K} f(x, h(x)) d x
$$

and the investigation of continuity properties of the function $\hat{h} \rightarrow I(\hat{h})$. To do that, obviously, one needs to know certain properties of the dependence of sets $\mathcal{M}(\hat{h})$ on $\hat{h}$.

In Sections 2 and 3 we shall show the following results.

Theorem 1. Let $Q \subset \mathbb{R}^{n}$ be bounded Lipschitz domain and let the set $M \subset \mathbb{R}^{m}$ is bounded and closed. Then for every given sequences $\left\{\hat{h}_{k}\right\}$ and $\left\{h_{k}\right\}$ such that

(i) $\left\{\hat{h}_{k}\right\} \subset$ coM and $\hat{h}_{k} \rightarrow \hat{h}_{0}$ in $\mathbb{R}^{m}$ as $k \rightarrow \infty$;

(ii) $h_{k} \in \mathcal{M}\left(\hat{h}_{k}\right), \quad k=1, \ldots$,

there exists a sequence $\left\{h_{0 k}\right\} \subset \mathcal{M}\left(\hat{h}_{0}\right)$ such that

$$
h_{k}-h_{0 k} \rightarrow 0 \text { strongly in } L_{2}\left(Q ; \mathbb{R}^{m}\right) \text { as } k \rightarrow \infty \text {. }
$$

Theorem 2. Let $Q \subset \mathbb{R}^{n}$ be bounded Lipschitz domain and let the set $M \subset \mathbb{R}^{m}$ is finite or $M$ is the closed convex hull of a finite set of elements. Then for every fixed sequence $\left\{\hat{h}_{k}\right\} \subset$ coM that converges in $\mathbb{R}^{m}$ to an element $\hat{h}_{0}$ and for every given element $h_{0} \in \mathcal{M}\left(\hat{h}_{0}\right)$ there exists a sequence $\left\{h_{k}\right\}, h_{k} \in \mathcal{M}\left(\hat{h}_{k}\right), k=$ $1,2, \ldots$, such that

$$
h_{k}-h_{0} \rightarrow 0 \text { strongly in } L_{2}\left(Q ; \mathbb{R}^{m}\right) \text { as } k \rightarrow \infty \text {. }
$$

Remark 1. Obviously, from Theorem 2 it follows immediately that the multivalued mapping $\hat{h} \rightarrow \mathcal{M}(\hat{h})$ is lower semicontinuous on $\operatorname{co} M$ (for the definition and properties of multivalued mappings we refer to Kuratowski [1]).

Remark 2. It is easy to see that under hypotheses of Theorem 1 the function $\hat{h} \rightarrow I(\hat{h})$ defined by (1.1) is lower semicontinuous provided that $f$ is Caratheodory function and that $f$ has a majorant $f_{0} \in L_{1}(Q)$ (we recall that the set $M$ is bounded). If, in addition, the hypotheses of Theorem 2 are satisfied, then the function $\hat{h} \rightarrow I(\hat{h})$ is continuous on $c o M$.

\section{Proof of Theorem 1}

In this Section, we give the proof of Theorem 1. Since all reasonings below do not depend on concrete properties of the reference domain $Q$, then, without loosing a generality, all proofs are given for the standard case $Q=K:=(0,1)^{n}$. Since the set $M$ is bounded and closed, then the convex hull coM of $M$ is 
closed too and all sets $\mathcal{M}(\hat{h})$ with $\hat{h} \in c o M$ are nonempty closed sets. In what follows, we shall use the notion of the relative interior $r i A$ for convex sets $A$ from Euclidean spaces, for instance rico $M$ stands for the relative interior of the convex hull of $M$. For the definition of $r i A$ and other notations and properties for convex sets we refer to Rockafellar [3]. Let $r_{0}$ be dimension of coM.

Step 1. Let $\hat{h}_{0} \in$ ricoM. Then there exists $d>0$ such that $\hat{h} \in \operatorname{rico} M$ whenever $\hat{h} \in \operatorname{coM}$ and $\left|\hat{h}-\hat{h}_{0}\right| \leq d$. Let us fix $\varepsilon>0,0<\varepsilon<d / 4$, and let $\left|\hat{h}-\hat{h}_{0}\right| \leq \varepsilon$. Then the element

$$
\hat{h}_{*}=\hat{h}+\frac{d}{\varepsilon}\left(\hat{h}_{0}-\hat{h}\right) \in \operatorname{rico} M
$$

Let $h \in \mathcal{M}(\hat{h}), h_{*} \in \mathcal{M}\left(\hat{h}_{*}\right)$ be arbitrary chosen elements. By virtue of Lyapunov's theorem on the range of vectorial measures for every $\lambda \in[0,1]$ there exists a measurable set $E_{\lambda} \subset K$ such that

$$
\left|E_{\lambda}\right|=\lambda, \quad \int_{E_{\lambda}} h(y) d y+\int_{K \backslash E_{\lambda}} h_{*}(y) d y=\lambda \hat{h}+(1-\lambda) \hat{h}_{*} .
$$

For a special choice $\lambda=\lambda_{0}=1-\varepsilon / d$ we define $h_{0}$ as

$$
h_{0}(\cdot)=\chi_{E_{\lambda_{0}}}(\cdot) h(\cdot)+\left(1-\chi_{E_{\lambda_{0}}}(\cdot)\right) h_{*}(\cdot)
$$

where $\chi_{E}$ denotes the characteristic function of the set $E$. By construction, $h_{0} \in \mathcal{M}\left(\hat{h}_{0}\right)$ and

$$
\int_{K}\left(h(y)-h_{0}(y)\right)^{2} d y=\int_{K \backslash E_{\lambda_{0}}}\left(h(y)-h_{0}(y)\right)^{2} d y \leq 4 c(M) \varepsilon / d,
$$

where $c(M)$ depends only on $M$. Thus, the assertion of Theorem 1 holds whenever $\hat{h}_{0} \in \operatorname{rico} M$.

Step 2. Let $\hat{h}_{0}$ does not belong to ricoM. Because ricoM is not empty (provided that $M$ consists of more than one element), then there exist a vector $a \in \mathbb{R}^{m}$ and a constant $c$ such that

$$
|a|=1, \quad\left\langle a, \hat{h}_{0}\right\rangle=c<\langle a, \hat{h}\rangle \quad \text { for all } \hat{h} \in \operatorname{rico} M .
$$

Without loosing generality, we can assume that $c=0$, otherwise we can use the transform $\hat{h} \mapsto \hat{h}-\hat{h}_{0}$.

Let $M_{1}=\{h \in M \mid\langle a, h\rangle=0\}$. Because the sets $M$ and $M_{1}$ are compact, then there exists a continuous function $\gamma, \gamma(t)=0$ if $t \leq 0, \gamma(t)>0$ if $t>0$, such that

$$
\left\langle a, h-\hat{h}_{0}\right\rangle \geq \gamma\left(\operatorname{dist}\left\{h ; M_{1}\right\}\right) \text { for all } h \in M .
$$

Without loosing generality, we can assume that the function $\gamma$ is convex, otherwise we can pass to the bipolar $\gamma^{* *}$, which has the desired properties. By construction, for nonnegative $\tau$ there exists the inverse function $\tau \rightarrow \gamma^{-1}(\tau)$, $\gamma^{-1}(\gamma(t))=t$ for $t \geq 0$, which is continuous and strictly increasing on $\{\tau \in$ 
$\mathbb{R} \mid \tau \geq 0\}$. Now, from (2.1) and convexity of $\gamma$ it follows that for every chosen $h \in \mathcal{M}$ there exists an element $h_{*}$,

$$
h_{*} \in \mathcal{M}_{1}=\left\{h \in L_{2}\left(K ; \mathbb{R}^{r}\right) \mid h(y) \in M_{1} \quad \text { a.e. } y \in K\right\},
$$

such that

$$
\begin{aligned}
\| h-h_{*} & \|_{L_{2}\left(K ; \mathbb{R}^{m}\right)}^{2} \leq c(m, M) \int_{K}\left|h(y)-h_{*}(y)\right| d y \\
& \leq c(m, M) \gamma^{-1}\left(\gamma\left(\int_{K}\left|h(y)-h_{*}(y)\right| d y\right)\right) \\
& \leq c(m, M) \gamma^{-1}\left(\int_{K} \gamma\left(\left|h(y)-h_{*}(y)\right|\right) d y\right) \\
& \leq c(m, M) \gamma^{-1}\left(\left|\int_{K} h(y) d y-\int_{K} h_{*}(y) d y\right|\right),
\end{aligned}
$$

where $c(m, M)$ depends only on $m$ and $M$. This way, for our situation with a fixed $\hat{h}_{0} \in \operatorname{coM}_{1}$, for every $\hat{h} \in \operatorname{coM}$ and arbitrary chosen $h \in \mathcal{M}(\hat{h})$ there exists a corresponding $h_{*} \in \mathcal{M}_{1}$ such that

$$
\left\|h-h_{*}\right\|_{L_{2}\left(K ; \mathbb{R}^{m}\right)}^{2} \leq c(m, M) \gamma^{-1}\left(\left|\hat{h}-\hat{h}_{0}\right|\right) .
$$

By construction,

$$
\int_{K} h_{*}(y) d y=\hat{h}_{*} \in \operatorname{coM}_{1},
$$

$\mathcal{M}\left(\hat{h}_{0}\right) \subset \mathcal{M}_{1}$ and the dimension of $c o M_{1}$ is less than $r_{0}$. From now on, we have to approximate the element $h_{*} \in \mathcal{M}\left(\hat{h}_{*}\right) \subset \mathcal{M}_{1}$ by elements from $\mathcal{M}\left(\hat{h}_{0}\right) \subset \mathcal{M}_{1}$, i.e. we have reduced the dimension $r_{0}$ of our problem to the problem with dimension less than or equal to $r_{0}-1$.

Step 3. To conclude our reasoning by induction over the dimension $r_{0}$ we have to prove our assertion for the case $r_{0}=1$. If $\hat{h}_{0} \in$ ricoM, then we apply reasoning from Step 1. If $\hat{h}_{0}$ does not belong to ricoM, then the set $M_{1}$ from the Step 2 consists of only one element $\hat{h}_{0}$ and the set $\mathcal{M}_{1}$ consists of one constant function $h_{0}(y)=\hat{h}_{0}$ a.e. $y \in K$. For this case we can apply the same reasoning as in Step 2, what gives the statement of Theorem for $r_{0}=1$.

\section{Proof of Theorem 2}

In this Section, we give the proof of Theorem 2. Let $M=\left\{\bar{h}_{1}, \ldots, \bar{h}_{N}\right\} \subset \mathbb{R}^{m}$. Let $H$ be $m \times N$ matrix with columns $\bar{h}_{1}, \ldots, \bar{h}_{N}$ respectively and let

$$
\Lambda:=\left\{\bar{\lambda} \in \mathbb{R}^{N} \mid \bar{\lambda}=\left(\lambda_{1}, \ldots, \lambda_{N}\right), \lambda_{j} \geq 0, j=1, \ldots, N ; \lambda_{1}+\cdots+\lambda_{N}=1\right\} .
$$

To a given vector-function $h \in \mathcal{M}$ (it has only $N$ admissible values from $M$ ) we can appoint an element $\bar{\lambda}$ whose components $\lambda_{j}$ represent the volume fractions 
in $K$ of the sets where the vector-function $h$ has the value $\bar{h}_{j}, j=1, \ldots, N$, respectively. Let $E:=\left\{\bar{z} \in \mathbb{R}^{N} \mid H \bar{z}=0\right\}$.

In these notations the statement of Theorem 2 is a straight consequence of:

$$
\left\{\begin{array}{l}
\text { if } \hat{\lambda}_{0} \in \Lambda,\left\{\bar{a}_{k}\right\} \subset \mathbb{R}^{N}, \bar{a}_{k} \rightarrow 0 \text { as } k \rightarrow \infty \\
\quad \text { and }\left\{\hat{\lambda}_{0}+\bar{a}_{k}+E\right\} \bigcap \Lambda \neq \emptyset, k=1,2, \ldots, \\
\text { then there exists a sequence }\left\{\bar{\lambda}_{k}\right\} \text { such that } \\
\bar{\lambda}_{k} \rightarrow \hat{\lambda}_{0} \text { as } k \rightarrow \infty \\
\bar{\lambda}_{k} \in\left\{\hat{\lambda}_{0}+\bar{a}_{k}+E\right\} \bigcap \Lambda, k=1,2, \ldots
\end{array}\right.
$$

Indeed, first of all we have to take care only about volume fractions of sets where the functions under consideration take the corresponding values $\bar{h}_{1}, \ldots, \bar{h}_{N}$ (we always can prearrange the corresponding sets preserving their measures). Further, for $h \in \mathcal{M}$ with corresponding volume fractions $\left(\lambda_{1}, \ldots, \lambda_{N}\right)=\bar{\lambda}$ we have that $h \in \mathcal{M}(H \bar{\lambda})$, and every $\hat{h} \in \operatorname{co} M$ has the representation $\hat{h}=$ $H(\hat{\lambda}+E)$ with some $\hat{\lambda} \in \Lambda$. The convergence $\bar{a}_{k} \rightarrow 0$ as $k \rightarrow \infty$ in (3.1) implies the corresponding convergence $\hat{h}_{k} \rightarrow \hat{h}_{0}$ in Theorem 2 , and the convergence $\bar{\lambda}_{k} \rightarrow \hat{\lambda}_{0}$ implies the corresponding convergence $h_{k} \rightarrow h_{0}$ in Theorem 2 .

Let us denote $\overline{1}=(1, \ldots, 1) \in \mathbb{R}^{N}$ and let us represent $E$ as the direct sum $E=E_{0} \oplus E_{1}$ where

$$
E_{0}=\{\bar{z} \in E \mid\langle\bar{z}, \overline{1}\rangle=0\} .
$$

Here the subspace $E_{1}$ can be equal to $\{0\}$ if $\overline{1}$ is orthogonal to $E$. From assumptions on $\bar{a}_{k}$ we have the existence of $\bar{z}_{0 k} \in E_{0}$ and $\bar{z}_{1 k} \in E_{1}$ such that

$$
\begin{aligned}
& \hat{\lambda}_{0}+\bar{a}_{k}=\bar{z}_{0 k}+\bar{z}_{1 k} \in \Lambda, \\
& \left\langle\bar{a}_{k}+\bar{z}_{1 k}, \overline{1}\right\rangle=0, \quad \bar{z}_{1 k} \rightarrow 0 \quad \text { as } \quad k \rightarrow \infty .
\end{aligned}
$$

So, if necessary, using the transform $\bar{a}_{k} \rightarrow \bar{a}_{k}+\bar{z}_{1 k}$ and replacing $E$ by $E_{0}$, without loosing generality, we can assume that

(i) the vector $\overline{1}$ is orthogonal to $E$;

(ii) $\left\langle\bar{a}_{k}, \overline{1}\right\rangle=0, k=1,2, \ldots$

That means (since $\left\langle\hat{\lambda}_{0}, \overline{1}\right\rangle=1$ ) our further reasoning concerns only the hyperplane $\left\{\bar{z} \in \mathbb{R}^{N} \mid\langle\overline{1}, \bar{z}\rangle=1\right\}$. There are two possibilities:

(a) $\left(\hat{\lambda}_{0}+E\right) \cap r i \Lambda \neq \emptyset$;

(b) $\hat{\lambda}_{0}$ belongs to a façade $\Lambda_{s}$ of $\Lambda$ with the dimension $s, 0 \leq s \leq N-2$, and $\hat{\lambda}_{0}+E$ can be separated from ri .

For the case $(a)$ there exists a $\lambda_{*} \in\left(\hat{\lambda}_{0}+E\right) \bigcap$ ri $\Lambda$ and the elements

$$
\lambda_{k}=\hat{\lambda}_{0}+\bar{a}_{k}+\tau_{k}\left(\bar{\lambda}_{*}-\hat{\lambda}_{0}\right), \quad k=1,2, \ldots,
$$

with appropriate $\tau_{k}>0, k=1,2, \ldots$, solve the problem for $k \geq k_{0}$ with some $k_{0}$. 
For the case $(b)$, without loosing generality, we can assume that $\Lambda_{s}$ is the façade with the minimal dimension $s$ compared to all façades, which contain $\hat{\lambda}_{0}$. Hence, after relabeling indexes we obtain

$$
\begin{aligned}
& \Lambda_{s}=\left\{\bar{\lambda} \in \Lambda \mid \bar{\lambda}=\left(\lambda_{1}, \ldots, \lambda_{N}\right), \lambda_{s+2}=\cdots=\lambda_{N}=0\right\}, \\
& \hat{\lambda}_{0}=\left(\lambda_{1}^{0}, \ldots, \lambda_{N}^{0}\right), \quad 0<\lambda_{1}^{0}, \ldots, 0<\lambda_{s+1}^{0}, \quad \lambda_{s+2}^{0}=\cdots=\lambda_{N}^{0}=0 .
\end{aligned}
$$

If

$$
\hat{\lambda}_{0}+\bar{a}_{k}+\bar{z}_{k} \in \Lambda \quad \& \quad \bar{z}_{k} \in E, k=1,2, \ldots
$$

then from

$$
\bar{a}_{k} \rightarrow 0 \quad \text { as } \quad k \rightarrow \infty, \quad \text { and } \quad\left\langle\bar{z}_{k}, \overline{1}\right\rangle=0, k=1,2, \ldots
$$

it follows immediately that the sequence $\left\{\bar{z}_{k}\right\}$ is bounded.

Let us assume that the sequence $\left\{\bar{z}_{k}\right\}$ converges to an element $\bar{z}_{0}$. If $\bar{z}_{0}=0$, then the sequence

$$
\bar{\lambda}_{k}:=\hat{\lambda}_{0}+\bar{a}_{k}+\bar{z}_{k}, k=1,2, \ldots
$$

solves the problem.

If $\bar{z}_{0} \neq 0$, then those entries of $\bar{z}_{0}=\left(z_{01}, \ldots, z_{0 N}\right)$, which are different from zero, are positive for $j \geq s+2$ and negative for those indexes $j^{\prime \prime}$, for which $\lambda_{j^{\prime \prime}}^{0}=1$ (if any). Therefore, there exists $d_{0}>0$ such that $\hat{\lambda}_{0}+\tau \bar{z}_{0} \in \Lambda$ provided $0 \leq \tau \leq d_{0}$.

Since $\bar{z}_{k}-\bar{z}_{0} \rightarrow 0$ as $k \rightarrow \infty$, then the elements

$$
\bar{\lambda}_{k}:=\hat{\lambda}_{0}+\tau_{k} \bar{z}_{0}+\left(\bar{z}_{k}-\bar{z}_{0}\right)+\bar{a}_{k}
$$

for $k \geq k_{0}$ and with appropriate $\tau_{k}, \tau_{k} \rightarrow 0$ as $k \rightarrow \infty$, belong to $\Lambda$. Indeed, since $\left\langle\bar{z}_{0}, \overline{1}\right\rangle=0,\left\langle\bar{a}_{k}, \overline{1}\right\rangle=0,\left\langle\bar{z}_{k}, \overline{1}\right\rangle=0, k=1,2, \ldots$, we have to check only inequalities

$$
\lambda_{k j} \geq 0, \quad j=1, \ldots, N ;, \quad k=k_{0}, k_{0}+1, \ldots
$$

(obviously, $\bar{\lambda}_{k} \rightarrow \hat{\lambda}_{0}$ as $k \rightarrow \infty$ ). For those indexes $\left\{j^{\prime}\right\}$, for which entries of $\bar{z}_{0}$ are equal to zero,

$$
\lambda_{j^{\prime}}^{0}+a_{k j^{\prime}}+\left(z_{k j^{\prime}}-z_{0 j^{\prime}}\right) \geq 0, \quad k=1,2, \ldots,
$$

(by the initial assumptions on the sequence $\left\{\bar{a}_{k}\right\}$ ), but for the rest of indexes $\left\{j^{\prime \prime}\right\}$ either

$$
1>\lambda_{j^{\prime \prime}}^{0}>0
$$

or

$$
\lambda_{j^{\prime \prime}}^{0}=1 \quad \& \quad z_{0 j^{\prime \prime}}<0
$$

what is sufficient for the existence of $\tau_{k}$ with desired properties.

The general case of an arbitrary sequence $\left\{\bar{z}_{k}\right\}$ is treated by standard reasoning by contradiction, i.e., we assume the contrary that there exist $d>0$ and a sequence of indexes $\left\{k^{\prime}\right\}$ such that the distance from $\hat{\lambda}_{0}$ to $\left\{\hat{\lambda}_{0}+\bar{a}_{k^{\prime}}+E\right\} \bigcap \Lambda$ 
is greater than $d$. After that we take an arbitrary subsequence of $\left\{\bar{a}_{k^{\prime}}\right\}$, for which the corresponding sequence $\left\{\bar{z}_{k^{\prime}}\right\}$ converges. The proof of the first part of Theorem 2 is completed.

Now, let $M$ be closed convex hull of a finite number of elements $\left\{h_{1}, \ldots, h_{N}\right\}$ and let

$$
\begin{aligned}
& S:=\left\{\sigma \in L_{2}\left(K ; \mathbb{R}^{N}\right) \mid \sigma=\left(\sigma_{1}, \ldots, \sigma_{N}\right), 0 \leq \sigma_{j}(x) \leq 1, j=1, \ldots, N ;\right. \\
& \left.\sum_{j=1}^{N} \sigma_{j}(x)=1 \text { a.e. } x \in K\right\} .
\end{aligned}
$$

Since the function

$$
(\sigma, x) \rightarrow\left(h(x)-\sum_{j=1}^{N} \sigma_{j} h_{j}\right)^{2}
$$

is a normal integrand on $\Lambda \times K$ ( for every fixed $h \in \mathcal{M})$, then every $h \in \mathcal{M}$ has the representation

$$
h(x)=\sum_{j=1}^{N} \sigma_{j}(x) h_{j} \quad \text { a.e. } \quad x \in K
$$

with some $\sigma \in S$. In turn, a subset of piecewise constant elements is dense in $S$ and sets $\mathcal{M}(\hat{h})$ have the same property.

This way, by using Cantor's diagonal process, we have that it is sufficient to show the existence of the approximating sequence $\left\{h_{k}\right\}$ for the case of a piecewise element $h_{0} \in \mathcal{M}\left(\hat{h}_{0}\right)$. Let $Q_{i} \subset K, i=1, \ldots, s$, are the sets where the function $h_{0}$ is constant and takes values $g_{1}, \ldots, g_{s}$ from $M$ respectively. Now, we define the set $\tilde{M}:=\left\{h_{1}, \ldots, h_{N}, g_{1}, \ldots, g_{s}\right\}$ and sets

$$
\tilde{\mathcal{M}}(\hat{h}):=\left\{h \text { measurable in } K \mid h(x) \in \tilde{M} \text { a.e. } x \in K, \int_{K} h(x) d x=\hat{h}\right\} .
$$

By construction, $c o \tilde{M}=M$ and $\tilde{\mathcal{M}}(\hat{h}) \subset \mathcal{M}(\hat{h}) \forall \hat{h} \in M$.

If $\left\{\hat{h}_{k}\right\} \subset M$ and $\hat{h}_{k} \rightarrow \hat{h}_{0}$ as $k \rightarrow \infty$ then also $\left\{\hat{h}_{k}\right\} \subset \operatorname{co} \tilde{M}, \hat{h}_{0} \in \operatorname{co} \tilde{M}$ and $h_{0} \in \tilde{\mathcal{M}}\left(\hat{h}_{0}\right)$. This way, the existence of the desired approximating sequence $\left\{h_{k}\right\}$ follows immediately from the proof of the first part of Theorem 2. The proof of Theorem 2 is completed.

We conclude with a simple example, which shows that the statement of Theorem 2 is not, in general, true under hypotheses of Theorem 1. Let

$$
M=\left\{(-1,0,0),(1,0,0),\left(0, t, t^{2}\right), 0 \leq t \leq 1\right\} \subset \mathbb{R}^{3} .
$$

By construction,

$$
\begin{gathered}
\mathcal{M}\left(\left(0, t, t^{2}\right)\right)=\left\{\left(h_{1}, h_{2}, h_{3}\right) \in L_{2}\left(K ; \mathbb{R}^{3}\right) \mid\right. \\
\left.h_{1}(x)=0, h_{2}(x)=t, h_{3}(x)=t^{2} ; x \in K\right\} \quad \text { for } 0<t<1, \\
\mathcal{M}((0,0,0))=\left\{\left(h_{1}, h_{2}, h_{3}\right) \in L_{2}\left(K ; \mathbb{R}^{3}\right) \mid h_{1}(x)=-1 \text { or } 0 \text { or } 1,\right. \\
\left.\int_{K} h_{1}(x) d x=0 ; h_{2}(x)=0, h_{3}(x)=0 ; x \in K\right\},
\end{gathered}
$$


and the statement of Theorem 2 does not hold.

\section{Acknowledgement}

This work has been partially supported by the project 05.1385 of the Latvian Council of Sciences.

\section{References}

[1] K. Kuratowski. Topology 1. Academic Press, New York London, 1966.

[2] G.W. Milton. The Theory of Composites. Cambridge University Press, Cambridge, 2002.

[3] R.T. Rockafellar. Convex Analysis. Princeton University Press,Princeton, 1970.

[4] J. Warga. Optimal Control of Differential and Functional Equations. Academic press, New York London, 1972. 